\title{
Planning with Strategic Goals
}

\author{
Evellin Cardoso \\ Jennifer Horkoff \\ University of Trento, Italy \\ Chalmers and the \\ Roberto Sebastiani \\ John Mylopoulos \\ University of Trento, Italy \\ University of Ottawa \\ evellin.souzacardoso@unitn.it University of Gothenburg, Sweden roberto.sebastiani@unitn.it jmylopou@eecs.uottawa.ca \\ jenho@chalmers.se
}

\begin{abstract}
Strategic goals and strategic planning have received much attention in Management Sciences literature since the 60s. In this work, we are interested in putting strategic planning on a formal, algorithmic footing by offering a formal reasoning technique for automatic generation and selection of strategic plans. Towards this end, in previous work [1] we have introduced the concept of strategic goals and dimensional refinement operators that define strategic goals in terms of domain dimensions from the data warehouses literature. Examples of dimensions for a strategic goal such as "Increase sales in Europe over 2 years" might include time, geography and product type. Here, we propose a formalization of strategic goals and their dimensional refinements that allows one to express a strategic goal model as a planning space that can be achieved across different dimensions. Subsequently, we use automated reasoning solvers to produce optimum strategic plans to achieve such strategic goals. Our proposal is illustrated with an example from the literature.
\end{abstract}

\section{INTRODUCTION}

Strategic goals and strategic planning have received much attention in the literature since the $60 \mathrm{~s}$, as they are concerned with long-term, global objectives of an organization and the strategies through which these are achieved. Many techniques for planning have been proposed [2], and there are commercial software products that support some of them [3].

Although much research has been devoted to various forms of strategic planning, little automatic support is provided for the systematic generation and selection of strategic plans from strategic goals. For example, approaches in Management literature [2], [4] explicitly deal with goal planning, but goals are usually specified using natural language and thus, no formal support for the generation of plans is provided. Goal-Oriented Requirements Engineering (GORE) goes further by capturing stakeholder interests using goals as abstraction. Many GORE techniques can estimate the level of satisfaction of top goals on the basis of different system designs [5], [6] and recommending specific system designs [7]. However, GORE techniques on software engineering are not able to represent and reason with essential enterprise properties in goal models (e.g. company's geographical distribution and its different products/services).

Enterprise Modeling (EM) techniques [8], [9] borrow the GORE concept of goal and thus, such techniques are not able to explore different alternatives to achieve strategic goals on the basis of such enterprise properties (e.g., sales can be increased differently in different locations where the company operates). To tackle this problem, in previous work we provided the SIENA modeling framework [1] that distinguishes among goals of various shades (mission, vision, strategic goals,

978-1-4673-6630-4/15/\$31.00 (c) 2015 IEEE etc.) and allows modelers to refine strategic goals in terms of dimensional refinement operators.

In this paper, we are interested in putting strategic planning on a formal, algorithmic footing by proposing an approach that generates and automatically selects strategic plans to achieve strategic goals. Our technique starts with the formalization of strategic goals from the SIENA framework which are defined in terms of different enterprise dimensions, inspired by data warehouse literature [10]. Then, it generates different alternatives (strategic plans) for strategic goal achievement based on enterprise dimensions (e.g., sales can be increased differently in different locations by promotions or new sales channels). Finally, the approach selects optimum strategic plans with respect to some objective function, taking into account the probability of occurrence of certain (un)desired business scenarios. Our research baseline includes strategic goals from the SIENA modeling framework and formal reasoning techniques for goal models [5], [6], [7], more specifically, the Constrained Goal Models (CGM) formalism [7].

The rest of the paper is structured as follows: Section II provides the research baseline for our work that includes the SIENA framework and the CGM formalism. Section III depicts an approach for the generation of strategic plans by formalizing strategic goals, dimensional refinements and optimization goals, whereas Section IV presents the mapping of strategic planning concepts into the CGM formalism. Section V shows one illustrative example of the generation of optimum strategic plans using the CGM tool, whereas Section VI contrasts our approach with related work. Finally, Section VII concludes the paper and outlines directions for future work.

\section{RESEARCH BASELINE}

Our research baseline consists of the SIENA Modeling Framework for enterprise modeling and the Constrained Goal Models (CGM) formalism.

SIENA Modeling Framework. The SIENA modeling framework [1] provides a conceptual foundation that distinguishes among goals of various shades (missions, strategic goals, etc.) and ontologies of processes/operations in enterprise modeling. Although the framework provides a rich ontology of goalrelated concepts, we particularly focus on the concepts required for our strategic planning approach. We illustrate such concepts using the example from a metal company [4, p.222] extracted from Management literature.

In SIENA, strategic goals reflect organization's strategy to achieve success in business. They are said to be global as the entire organization is responsible for their achievement. 
To characterize such variability, the framework introduces refinement dimensions. Refinement dimensions correspond to different properties along which goals can be characterized such as location, time or product types properties. For example, a refinement over the location dimension of the "Increase sales over 3 years" strategic goal (with the country granularity) yields the "Increase sales in Germany over 3 years", "Increase sales in France over 3 years" and "Increase sales in Italy over 3 years" sub-goals. Other refinement of the same goal across the time dimension (within the year granularity) could also be performed, yielding the "Increase sales over 1st year", "Increase sales over 2nd year" and "Increase sales over 3rd year" strategic sub-goals.

A direct consequence of refining strategic goals in terms of refinement dimensions is the ability to specify different "solutions" ("tactics" or tactical goals) for implementing the same strategic goal along different points of interest of a dimension. Following in the example, it is possible not only to specify the alternatives for increasing sales for a given company (e.g., increase sales by promotions or by opening new sales channel), but one can also explore the alternatives for increasing sales depending on the country the company operates. For that, implements relations from the tactical goal to the strategic goal are used to denote that one tactics implements a given strategic goal. Once it is possible to schedule the achievement of tactical goals, an operation is in charge of planning the execution of each tactics.

Besides goals and their decompositions, managers are also interested in foreseeing aspects that may influence the fulfillment of strategic goals. In that respect, SIENA proposes to model SWOT factors [11] (Strengths, Weaknesses, Opportunities and Threats) in terms of the concept of situation. A situation characterizes a state of affairs (state of the world) in terms of the entities that exist in that state, their properties and interrelations. Favorable situations are represented via positive influence links on goals, whereas unfavorable situations are represented via negative influence links.

Although SIENA proposes a rich characterization of goals, the framework refrains from using such characterization to automatically perform strategic planning for the achievement of strategic goals. In order to achieve such intent, as SIENA strategic goals have no formal semantics in its current version, we need to either assign our own formal semantics for reasoning or find existing goal-modeling languages with already wellestablished semantics for reasoning. In particular, we opted for the second choice by choosing the Constrained Goal Models (CGM) formalism described in the following.

The Constrained Goal Models (CGM) Formalism. The CGM formalism [7] consists of a modeling and automatedreasoning suite for decomposing stakeholders' goals as an AND/OR graph of alternative refinements and relations, and for automatically finding the optimum set of sub-goals according to a combination of objective functions.

Regarding CGM modeling functionalities, goal and their relations are represented in terms of CGM nodes, CGM refinements and CGM relation edges. Starting with CGM nodes, CGM considers stakeholders' requirements as desired state of affairs that the system has to achieve. Such requirements (root goals) need to be progressively refined into intermediate goals until producing tasks (actionable/leaf goals) that can be executed. Users' assertions enables the specification of optional requirements that would be interesting to be fulfilled in the case they are not conflicting with other requirements. Regarding CGM relation edges, each non-leaf goal can be OR-decomposed into one of its alternative incoming goal refinements or further AND-decomposed into a conjunction of sub-goals necessary to achieve it. Contributions between goals can be represented by means of contribution edges $\left(\mathrm{G}_{i} \stackrel{++}{\longrightarrow}\right.$ $\mathrm{G}_{j}$ ) that express that if the source goal $\mathrm{G}_{i}$ is satisfied, then the target goal $\mathrm{G}_{j}$ must be satisfied (but not vice versa).

Besides graphical constraints expressed as relation edges, goal models can be enriched with numerical constraints on goals and refinements to express user-defined constraints among nodes. For example, if one estimates that goal $G_{1}$ costs $80 €$ to be achieved and goal $G_{2}$ costs $200 €$, one can use such information to write constraints in terms of costs among goals $G_{1}$ and $G_{2}$. Finally, a singular or combination of objective functions to optimize (i.e. maximize or minimize) can also be specified as functions of boolean and numerical variables.

Regarding CGM reasoning functionalities, once goals are modeled and objective functions are specified accordingly, stakeholders may request the CGM solver to automatically generate realization(s) that correspond to one of the alternative ways of refining the mandatory requirements (and potentially some of the optional ones) in compliance with the user's assertions and constraints. The CGM solver then generates realizations that optimize one single objective or a lexicographically ordered combination of objectives.

CGMs is supported by the CGM-tool [12], which is implemented as a standalone java application based on the Eclipse RCP engine. CGM-tool uses a state-of-the-art Optimization Modulo Theories (OMT) solver [13], [14] as backend automated-reasoning engine. The tool provides functionalities to create CGM models as graphical diagrams and to perform different forms of reasoning, including interactive search for realizations. As CGM's advanced functionalities in the generation of optimum realizations are comparable to the generation of alternative strategic plans in strategic planning, we here use the CGM-tool as backend for strategic planning aimed at generating optimum strategic plans.

\section{The Strategic Planning ApProACH}

This section presents the strategic planning approach for the generation of strategic plans to achieve strategic goals. The approach is presented by means of the formalization of strategic goals, their AND/OR/d-refinements and implement relations introduced in $\mathrm{Sec}$. II.

Strategic Goals Elaboration and Refinement. Once strategic goals are elaborated (e.g. "Increase sales over 3 years", Sec. II), the next step consists of planning their achievement with company's capabilities and resources. For that, they need to be refined and operationalized.

In order to present the semantic of refinements, a strategic goal is formally represented as a parameterized goal with one parameter per dimension. For example, the "Increase sales by $2 \%$ over 3 years" goal is represented as IncrSales(3YR, Europe, AllProducts), where 3YR, Europe, AllProducts are 
respectively the parameters of the three dimensions spanned by the goal. In order to define the numeric increase of $2 \%$, we need to express the increase of $2 \%$ in sales for all products throughout Europe over three years. For this, we assume an indicator "sales" associated with each IncrSales goal and define

IncrSales $(3 Y R$, Europe, All Products $)=$ $=0.02 *$ sales (now, Europe, AllProducts)

As the dimensional refinement (d-refinement) conjunctively refines a strategic goal along a dimension, we also need to determine how the global sales increase of $2 \%$ is distributed along the subgoals. For example, IncrSales might be refined along the LOCATION dimension with specific targets for sales for each country in Europe, or a subset thereof since there might not be ambitions for certain countries. In order to determine targets for the subgoals of a d-refinement, we want the targets to be based on real past data. As such, we use a criterion to set metrics for each target calculation for each dimensional subregions over which we are refining. This criterion is selected by managers following his/her preferences. For example, for IncrSales, suppose we are interested in increasing sales in only three countries (Germany, France and Italy) and use the growth of the economy last year of each country ("growth") as criterion to estimate the targets for each country. Going forward with the first criterion, then:

IncrSales $(3 Y$ R, Europe, AllProducts $)=$

$$
\begin{array}{r}
\text { IncrSales }(3 Y R, \text { Germany, AllProducts })+ \\
\text { IncrSales }(3 Y R, \text { France, AllProducts })+ \\
\text { IncrSales }(3 Y \text { R, Italy, AllProducts })
\end{array}
$$

where

$$
\begin{aligned}
& \text { IncrSales }(3 Y \text { R, Germany, AllProducts })= \\
& =\frac{\operatorname{growth}(\text { Germany })}{\operatorname{growth}(\text { Germany })+\operatorname{growth}(\text { France })+\operatorname{growth}(\text { Italy })} * \\
& x * \text { sales (now, Germany, AllProducts) }
\end{aligned}
$$

and similarly for France and Italy. Here, the second line of equation (3) corresponds to the normalization factor that allocates to each country a sales growth normalized by the size of economy growth of the corresponding country last year, whereas the $\mathrm{x}$ factor in third line corresponds to the percentage sales increase in the country.

By combining equations (1), (2) and (3) (and similarly for France and Italy), the actual growth of economy last year ("growth") criterion: $\operatorname{growth}($ Germany) $=2 \%, \operatorname{growth}($ France $)=1 \%$ and $\operatorname{growth}($ Italy $)$ $=0.5 \%$ and current sales: sales (now, Germany, AllProducts) $=10000$, sales(now, France, AllProducts) $=7000$ and sales(now, Italy, AllProducts) $=7000$, we find $x=1.57 \%$ (rounded). This yields a targeted increase of $3.14 \%$ in sales in Germany, a targeted increase of $1.57 \%$ in France and a targeted increase of $0.78 \%$ in Italy. Currently, although these numbers are manually acquired together with their corresponding calculations, we intend to automatically generate goal refinements and check their consistency. Fig. 1(b) shows this refinement by LOCATION with their corresponding targeted increase for each country.

Orthogonal to this d-refinement, one may want to d-refine IncrSales along the TIME dimension using expected growth of the economy of each country ("expGrowth") as criterion in the compound interest equation below:

$$
\begin{aligned}
& \text { IncrSales }(3 Y R, \text { Europe, AllProducts })= \\
& \quad \text { sales }(\text { now, Europe, AllProducts }) *(1+\text { expGrowth })^{3}
\end{aligned}
$$

In this case, as the total current sales is sales(now, Europe, AllProducts) $=24000$ (from previous example) and we have a targeted increase of $2 \%$ in the total sales, we have calculated using equation (4) an uniform expected growth of economy (expGrowth) for each year whose value is $0.6622 \%$ (i.e., $\operatorname{expGrowth}(Y R 1)=\operatorname{expGrowth}(Y R 2)=\operatorname{expGrowth}(Y R 3)=$ $0.6622 \%)$. Fig. 1(c) shows the refinement by TIME with its corresponding targets for sub-goals.

As presented in Sec. II, besides d-refinements, strategic goals can also be AND/OR-refined and implemented by tactical goals. Therefore, the strategic planning approach has to accommodate d-refinements and AND/OR/implement relations for strategic goals. In SIENA, by d-refining a strategic goal $\mathrm{G}(\mathrm{X}, \mathrm{Y}, \mathrm{Z})$ into $\mathrm{G}_{1}\left(\mathrm{X}_{1}, \mathrm{Y}_{1}, \mathrm{Z}_{1}\right), \ldots, \mathrm{G}_{N}\left(\mathrm{X}_{N}, \mathrm{Y}_{N}, \mathrm{Z}_{N}\right)$ and also AND-refining/OR-refining/implementing the same goal into $\mathrm{G}_{1}{ }_{1}, \mathrm{G}_{2}, \ldots, \mathrm{G}_{M},{ }$, the second refinement (i.e., $\mathrm{G}_{1}{ }_{1}, \mathrm{G}_{2}$, $\left.\ldots, \mathrm{G}_{M}{ }_{M}\right)$ inheres by default the properties of $\mathrm{G}_{1}\left(\mathrm{X}_{1}, \mathrm{Y}_{1}, \mathrm{Z}_{1}\right), \ldots$ , $\mathrm{G}_{N}\left(\mathrm{X}_{N}, \mathrm{Y}_{N}, \mathrm{Z}_{N}\right)$. More concretely, if we d-refine IncrSales into $\mathrm{G}_{1}=$ "Increase sales by $0.6622 \%$ in year 1 ", $\mathrm{G}_{2}=$ "Increase sales by $0.6622 \%$ in year 2 ", $\mathrm{G}_{3}=$ "Increase sales by $0.6622 \%$ in year 3", and also implement it into using (tactical goals) $G_{1}=$ "Increase sales by opening new sales channels", $\mathrm{G}_{2}{ }_{2}=$ "Increase sales by training sales staff", $\mathrm{G}_{3}$ = "Increase sales through promotions", then the sub-goals $\mathrm{G}_{1}$, $\mathrm{G}_{2}{ }_{2}$ and $\mathrm{G}_{3}{ }_{3}$ inheres the properties of $\mathrm{G}_{1}, \mathrm{G}_{2}, \mathrm{G}_{3}$, unless the analyst chooses to override it. In this particular case, $\mathrm{G}_{1}{ }_{1}, \mathrm{G}_{2}{ }_{2}$ and $\mathrm{G}_{3}$ inheres the property "year 1 ", "year 2" and "year 3" from $G_{1}, G_{2}$ and $G_{3}$, respectively. Fig. 1(c) depicts this example of refinements inheritance with tactical goals inhering the properties of strategic goals. Further, the analyst chose to prune some tactical goals, thus applying a specific tactics for each year due to trends revealed by past real data. Therefore, "Increase sales in year 2 by opening new sales channels" and "Increase sales in year 3 by opening new sales channels" have been pruned (and the other tactics for the other years.)

The same rationale applies if two orthogonal d-refinements are performed successively, i.e., the subgoals of d-refinement1 inheres the properties of d-refinement 2 and vice versa. Obviously, inheritance of a refinement includes the criterion associated with the refinement. For instance, since expGrowth is the criterion for the TIME d-refinement above, when it is inherited by IncrSales(3YR, Germany, AllProducts), IncrSales(3YR, France, AllProducts), IncrSales(3YR, Italy, AllProducts) respectively, it will generate three subgoals for each one of them and use expGrowth to normalize targets for these subgoals. Fig. 1(d) illustrates the result of two successive drefinements of "Increase sales by $2 \%$ over 3 years". In the first d-refinement by LOCATION, equations (2) and (3) are used with the respective growth criteria, thus generating the following sub-goals $\mathrm{G}_{1}=$ "Increase sales in Germany by $3.14 \%$ over 3 years", $\mathrm{G}_{2}=$ "Increase sales in France by $1.57 \%$ over 3 years", $\mathrm{G}_{3}=$ "Increase sales in Italy by $0.78 \%$ over 3 years". In the second d-refinement by TIME, the d-refinement inherited the growth criterion from the first d-refinement and yielded the nine sub-goals depicted in Fig. 1(d). Observe also that contrasting with previous example (Fig. 1(c)) in which some goals have been pruned based on trends of past real data, the two successive d-refinements here yielded nine sub-goals.

The refinement process (with defaults) can end when a given strategic goal has reached leaf elements for all points of interest of the dimensions spanned by the goal, or when this strategic goal spans regions that are sufficiently uniform so that they do not require further refinements. For example, if Italy is deemed sufficiently uniform to admit one tactical solution 


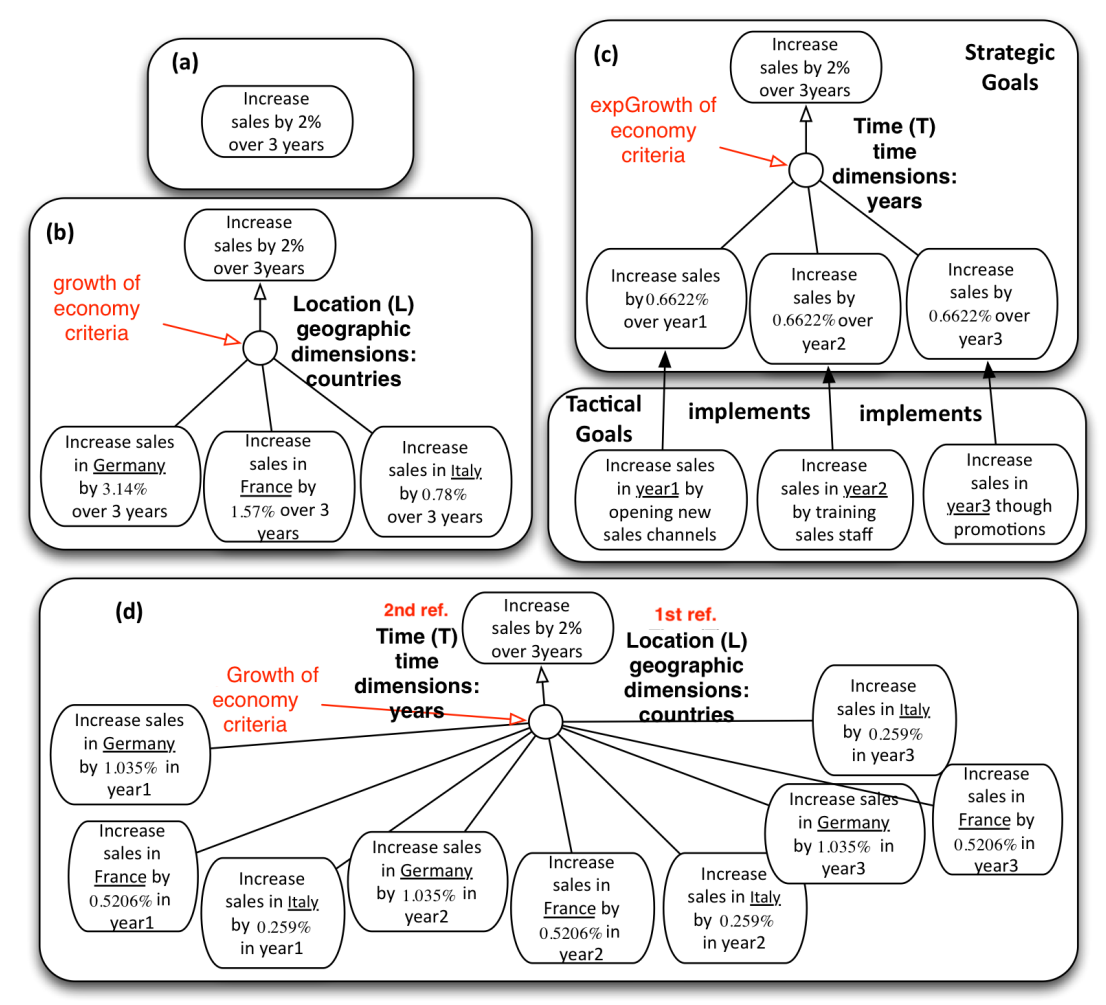

Fig. 1. Strategic Goals, Dimensional Refinements and Properties Heritage

for all its subregions (provinces and stores)(e.g. "Train sales staff"), then the analyst do not need to drill further down in the planning.

Scenario Analysis. In order to represent how aspects of business may impact the achievement of strategic goals, the SIENA framework provides the concept of situation (Sec. II). In a strategic planning approach, as it is usually difficult to foresee how isolated situations may affect goals, managers are rather interested in determining how the whole business environment might evolve, specially in the presence of complex or rapid changes. In order to cope with uncertainty, managers usually carry out scenarios analyses by building detailed and plausible views (scenarios) about how the business environment of the company might develop in the future [4]. In our approach, scenarios are represented as a set of situations $\left\{s_{1}, s_{2}, \ldots, s_{n}\right\}$ that represent the company context similarly in [9]. Scenario analysis is carried out by marking the situations $\left\{s_{1}, s_{2}, \ldots\right.$, $\left.s_{n}\right\}$ of the corresponding scenario as true to indicate they are active in a given analysis.

Strategic Plans. Once strategic goals have been specified and situation analysis has ended, strategic planning concepts (strategic, tactical goals, operations and situations) have been specified accordingly. The outcome of this phase consists of a strategic goal model (planning space) with several alternatives strategic plans to achieve its strategic goals. Fig. 2 depicts a schematic representation of this outcome, by depicting a model with the strategic planning concepts, one active scenario with its corresponding situations marked as true (represented as red ticks) and the two possible strategic plans (a blue and red strategic plans) that may achieve the top strategic goal. In this context, our strategic planning approach is interested in:
How to automatically find different ways of satisfying strategic goals, i.e., different strategic plans?

Optimization Goals. In order to differentiate among strategic plans, assigning them quantitative values and thus enabling the ranking of strategic plans, the strategic planning approach also includes optimization goals. For example, we may be interested in a strategic plan for achieving IncrSales that minimizes expenses, or maximizes profits. We express such goals as

$$
\text { OPT [cost, IncrSales (3Y R, Europe, AllProducts)] }
$$

OR

OPT [profit, IncrSales (3YR, Europe, AllProducts)] (6)

Therefore, each optimization goal is defined relative to an attribute (or a linear combination thereof) and a strategic goal. Note that in order to generate optimum plans, we need to have values for these attributes for every tactical goal used to realize a strategic goal for all leaf-level regions. For our example, this means that we know (estimated) costs and profits for the operations of our model. Such costs and profits are respectively represented as $C_{1}, P_{1}, \ldots, C_{4}, P_{4}$ in Fig. 2.

Once the strategic planning approach produced a number of strategic plans that can be ranked in terms of different attributes, they need to be automatically selected according to the pre-specified optimization goals. As SIENA constructs have no formal semantics (Sec. II), we take advantage of CGM welldefined semantics for goal reasoning to automatically select realizations. In order to take such advantage, we need to map SIENA strategic planning concepts to the CGM formalism. This mapping is depicted in the next section. 


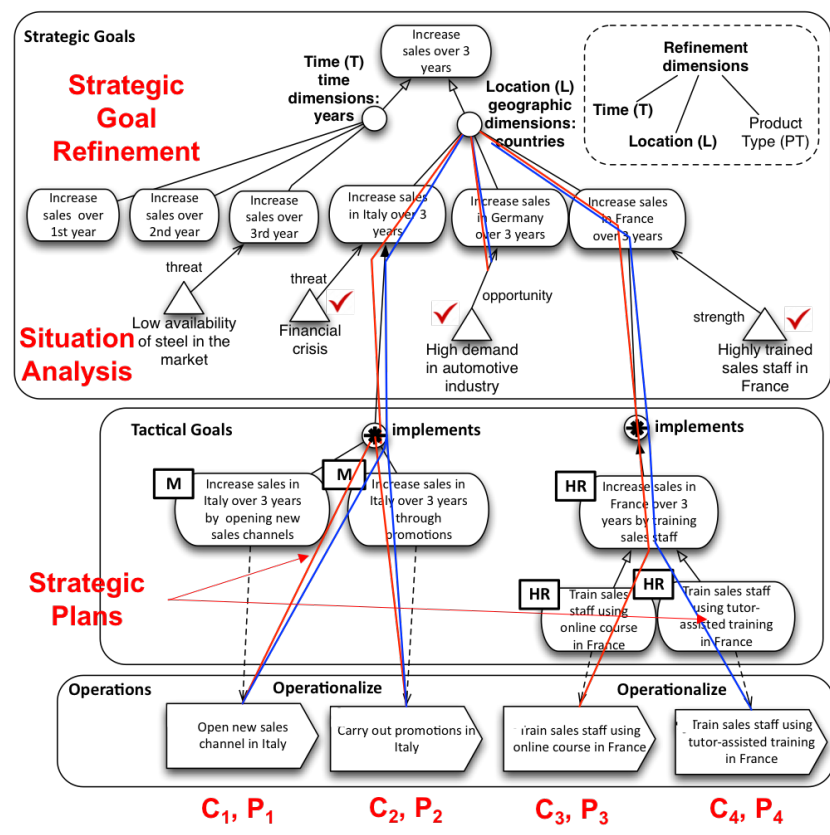

Fig. 2. Strategic Plans (Schematic Representation)

\section{Formal Reasoning With Strategic GoAls}

This section describes the approach for specifying SIENA's strategic planning concepts (Sec. II and III) using the CGM formalism. This specification enables us to automatically select optimum strategic plans for achieving strategic goal models.

\section{A. Specify Strategic Planning Concepts into CGM}

The approach starts by mapping the concepts mentioned in Sec. II and III to the CGM formalism with the purpose of providing formal CGM semantics for such modeling constructs.

Strategic, Tactical Goals, AND/OR/d-refinements, Implements Relation. In Sec. III, the strategic planning process starts with the specification of strategic goals and their subsequent refinements in terms of AND/OR/d-refinements until finding tactical goals that implement points of each dimension. This idea is very similar to the progressive refinement of the CGM root goal into intermediate goals and therefore, every top strategic goal is specified as a CGM root goal, while its strategic sub-goals and tactical goals correspond to intermediate goals in CGM. Both in SIENA and CGM, the process of goal refinement ends when no decomposition is required and the goal can be executed. Therefore, operations in SIENA correspond to a CGM task as both concepts refer to the lowest level of refinement in a goal tree. Regarding relations among goals, AND/d-refinements are mapped into one CGM refinement, while alternative AND/d-refinements $(\mathrm{OR})$ of the same strategic goal are mapped into multiple CGM refinements. Each implement relations is mapped into a CGM refinement to depict that a strategic goal is implemented by a conjunction of different tactics.

Situations and SWOT Relations. As situations are not part of any refinement tree in SIENA, they have been mapped to CGM root goals since root goals are also the most essential level of a refinement tree (although root goals are subject to further refinement in CGM, in the case of this mapping, the modeler should not refine such root goals). Concerning the SWOT relations that situations may have towards goals, strengths, opportunities, weaknesses and threats relations are represented by means of CGM contribution edges of situations towards goals. As the semantics of contribution edges states that, if the source goal is satisfied then the target goal must be also satisfied, this semantics is used to interpret that if one situation is included in a given analysis, the goals targeted by that situation should be also in the realization.

In order to represent scenarios in CGM, we mark the situations $\left\{s_{1}, s_{2}, \ldots, s_{n}\right\}$ of the corresponding scenario as true by means of CGM user's assertions to indicate they are active in a given analysis. For the situations that pertain to other scenarios, no further users' assertions have to be performed.

\section{B. Specify Optimization Goals as Objective Functions in CGM}

In the discussion about optimization goals (Sec. III), we argued that one may want to find optimum plans with respect to different attributes, e.g. strategic plans that minimizes costs or maximize profits. Therefore, we need to assign some quantitative values for the attributes of each strategic plan so that we can define the notion of optimum strategic plan. In our case, as CGM requires modelers to assign values to leaf goals, our strategic planning activity assigns costs and workingTime (duration) to operations to select the optimum realization.

In addition to objective functions, we may also elaborate on the constraints our strategic plan is subject to. Constraints may be of several types like technical, physical, environmental and stem from a variety of sources, such as limited resources, contractual obligations, particularities of the domain, etc. In our case, we defined ranges for costs of operations (e.g. 1000 $>$ cost $>200$ ) (in millions) and time limits (workingTime $<$ 8) (in years) for operations execution (depicted in Fig. 3(a)).

\section{ILlustrative EXAMPle}

In order to illustrate our strategic planning approach, Fig. 3 shows a strategic goal model resulted from the application of the mapping rules described in Sec. IV enriched with constraints and numerical values. With this strategic goal model in hands, our intention is to illustrate our strategic planning approach with the generation of different strategic plans on the basis of strategic goals.

In order to depict the generation of strategic plans, the strategic goal model has been enriched with the constraints depicted on top left of Fig. 3, numeric values depicted below operations in the same figure and a set of situations $s_{1}, \ldots$, $s_{6}$. With this set of situations, we simulated a scenario of financial crisis (scenario1) that should be active during the generation of strategic plan by marking $s_{2}=$ "Sudden spike of metal cost", $s_{5}=$ "Low demand of steel in market", $s_{6}=$ "Economic downturn" as true by means of user's assertions. Fig. 3(a) shows the strategic goal model with constraints, active situations (circled in red) and the generated strategic plan (in red). Tests with scenario analysis have been useful for the selection of plans in face of potential future scenarios (e.g. financial crisis scenario). Scenarios have been useful also for the selection of plans when there is more than one admissible plan according to the constraints. For example, with 
the constraints of Fig. 3(a), two plans are eliminated (one plan does not satisfy cost constraint, other does not satisfy the workingTime constraint and the third one does not satisfy the minimize(cost) objective function). In face of that, there are two possible plans. By marking $s_{2}, s_{5}, s_{6}$ as mandatory, the solver has just one plan (depicted in Fig. 3(a) in red).

Finally, when there exist multiple optimum strategic plans, the CGM solver does not depict all possible optimum plans, but rather just one admissible plan. Since there are potentially an exponential number of optimum solutions, the graphical representation of multiple optimum plans would not make sense. Therefore, our approach is bounded by such aspect of CGM to depict just one optimum strategic plan.

\section{RELATED WORK}

Much research has been dedicated to goal modeling and reasoning in different areas [5]. In this section, we review works related to our conceptualization of strategic goals together with reasoning approaches for strategic planning.

Management Literature. In Management Sciences, Planning corresponds to one of the core management activities, as it is related with the definition of organization's goals, followed by the corresponding operations and resource requirements necessary to achieve such goals [4]. Although Management literature provides a vast characterization of strategic goals in terms of their global nature, works such as Management by Objectives and Vision statements [2], [4] mainly specify goals by means of natural language, thus lacking formal rigor in the representation of modeling concepts. Consequently, reasoning techniques cannot be built in order to analyze goal models.

GORE Approaches. In GORE approaches, goals represent stakeholders' motivations for a target software system and goal models are used as the starting point for the generation of system requirements. In this context, a number of GORE reasoning techniques quantify the level of satisfaction of top system goals depending on alternative system designs [5], [15]. Other approaches go beyond as they can not only quantify the level of satisfaction of top system goals, but can also recommend which designs to select [6], [7]. In particular, the CGM approach used in this paper is able to find the optimum set of subgoals to achieve a given root goal. Although GORE techniques allow one to perform advanced reasoning with goal models, their scope relies on the evaluation/generation of system designs, not strategic plans.

Enterprise Modeling Approaches. Although the idea of variability is explored in RE, enterprise modeling approaches like BIM [8], [9] and ArchiMate [16] allow one to define strategic concerns in terms of goals, such as "Increase sales", but does not provide a modeling construct as our dimensional refinement operator. As a consequence, reasoning techniques cannot address enterprise variability as our planning approach.

AI planning. In AI planning, we focus on works that represent goal models graphically in terms of goals and their relationships (AND/OR and contribution links). In particular, Liaskos et al. [17] represent goal models in terms of goals, enriched with temporal constraints, optional goals, and preference goals. Then, the paper uses a planner to find a sequence of tasks which would satisfy all mandatory goals, respecting the precedence constraints. While those methods propose to apply planning for finding alternative system designs from goals models, our approach can be considered more expressive as our strategic goal models use dimensional refinement operators to express enterprise variability in goal models. Further, by using the CGM formalism, we also rely on a more expressive goal model formalism for the generation of different strategic plans.

\section{CONCLUSIONS AND Future WORK}

In this paper, we proposed a formal representation of strategic goals and an automated technique for the generation of optimum strategic plans to achieve such strategic goals on the basis of objective functions, constraints and scenarios. Starting with the notion of strategic goals and their refinement dimensions from our previous work [1], we propose a formalization of strategic goals, how to d-refine them in terms of refinement dimensions and how to accommodate d-refinements and other relations of strategic goals (AND-refinements and implement-relations). Once strategic goals have been formally specified, strategic planning concepts are mapped into the CGM formalism to generate optimum strategic plans on the basis of objective functions, constraints and scenarios.

Although our approach has advantages over traditional goal analysis, it still presents some limitations that we leave as future work. First, the numeric treatment for the distribution of weights of sub-goals is still very simplistic and requires an extensive work in the definition of rules for such distribution based on each type of refinement dimension (time, location, product/service). Second, as strategic goals refinements are manually performed, the approach lacks scalability in modeling if the size of the model grows. In order to cope of both shortcomings, in particular, we envision that the semantics of drill-down/roll-up operations from data warehouses [10] can be further explored for the automatic generation of goal refinements and for checking the consistency of such refinements. Third, although the refinement process of strategic goals in terms of their AND/OR/d-refinements deals with the determination of targets for sub-goals, this refinement process is solely used to produce the strategic goal model structure and the numeric targets of sub-goals are not used for the optimization solver to select among strategic plans. Therefore, such targets should be somehow incorporated into the CGM solver to enable the ranking of strategic plans based on targets.

We could have exploited more the functionalities of the CGM formalism by using more complex constraints. However, CGM limits the usage of constraints to only linear constraints (e.g. $\left.x^{*} a+y * b\right)$. Furthermore, as CGM only shows only one optimum solution per time, this is also a limiting factor for our approach. Further, scalability tests for our approach with larger goal models with a high number of constraints are also fundamental for the overall evaluation of our reasoning approach. Finally, we envision that similar ideas can be applied for the generation of tactical plans.

\section{REFERENCES}

[1] E. Cardoso, J. Mylopoulos, A. Mate, and J. Trujillo, "Strategic Enterprise Architectures," in Proc. of PoEM'16. Springer, 2016, pp. 57-71.

[2] D. Rigby and B. Bilodeau, "Management Tools \& Trends 2015 Survey," Bain \& Company, Tech. Rep., 2015. 


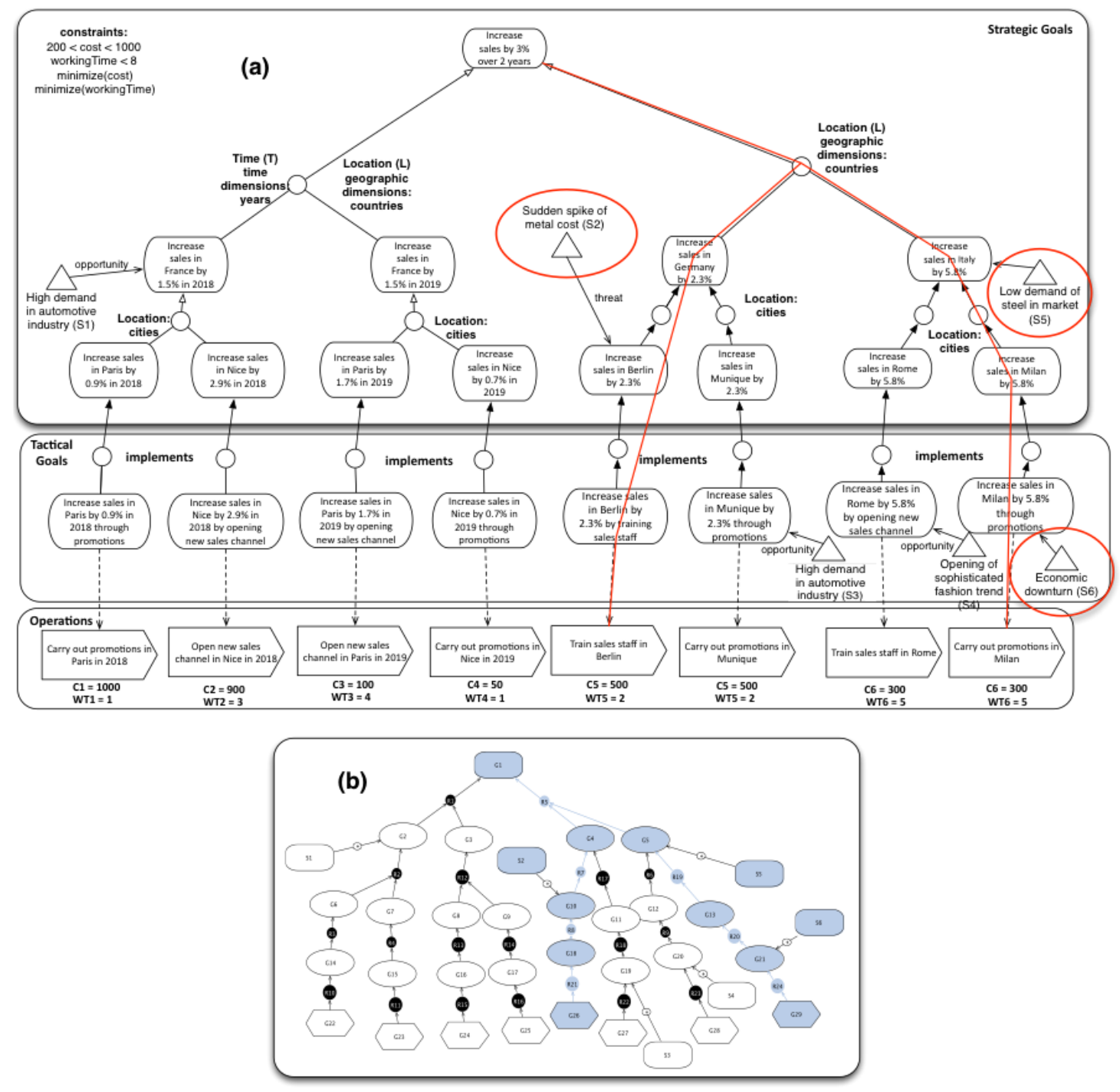

Fig. 3. Generation of alternatives strategic plans in CGM in a scenario of financial crisis

[3] K. S.R.O. (2000) BSC Designer. [Online]. Available: http://www.bscdesigner.com

[4] R. Daft and D. Samson, Fundamentals of Management: Asia Pacific Edition. Cengage Learning, 2014.

[5] P. Giorgini, J. Mylopoulos, E. Nicchiarelli, and R. Sebastiani, "Reasoning with Goal Models," in Proc. of ER'03. Springer, 2003, pp. 167-181.

[6] R. Sebastiani, P. Giorgini, and J. Mylopoulos, "Simple and MinimumCost Satisfiability for Goal Models," in Proc. of CAiSE'04. Springer, 2004, pp. 20-35.

[7] C. M. Nguyen, R. Sebastiani, P. Giorgini, and J. Mylopoulos, "MultiObjective Reasoning with Constrained Goal Models," Requirements Engineering, pp. 1-37, 2016.

[8] J. Horkoff, D. Barone, L. Jiang, E. Yu, D. Amyot, A. Borgida, and J. Mylopoulos, "Strategic Business Modeling: Representation and Reasoning," Software \& Systems Modeling, vol. 13, no. 3, pp. 10151041, 2014.

[9] A. Maté, J. Trujillo, and J. Mylopoulos, "Stress Testing Strategic Goals with SWOT Analysis," in Proc. of ER'15. Springer, 2015, pp. 65-78.

[10] N. Tryfona, F. Busborg, and J. G. Borch Christiansen, "starER: A Conceptual Model for Data Warehouse Design," in Proc. of Workshop on Data Warehousing and OLAP. ACM, 1999, pp. 3-8.

[11] T. Dealtry, Dynamic SWOT Analysis: Developer's Guide. Dynamic
SWOT Associates, 1992.

[12] D. Mekuria, C. M. Nguyen, R. Sebastiani, P. Giorgini, and J. Mylopoulos. (2015) CGM Tool. [Online]. Available: http://www.cgmtool.eu

[13] R. Sebastiani and S. Tomasi, "Optimization Modulo Theories with Linear Rational Costs," ACM Transactions on Computational Logics, vol. 16, no. 2, 2015.

[14] R. Sebastiani and P. Trentin, "OptiMathSAT: A Tool for Optimization Modulo Theories," in Proc. of CAV'15. Springer, 2015, pp. 447-454.

[15] W. Heaven and E. Letier, "Simulating and Optimising Design Decisions in Quantitative Goal Models," in Proc. of RE'11, 2011, pp. 79-88.

[16] C. Azevedo, J. Almeida, M. van Sinderen, and L. Ferreira Pires, "Towards Capturing Strategic Planning in EA," in Proc. of EDOC'15, 2015, pp. 159-168.

[17] S. Liaskos, S. A. McIlraith, S. Sohrabi, and J. Mylopoulos, "Representing and Reasoning About Preferences in Requirements Engineering," Requirements Engineering, vol. 16, no. 3, p. 227, 2011. 